\title{
Characterization of fractionated biomass component and recovered ionic liquid during repeated process of cholinium ionic liquid-assisted pretreatment and fractionation
}

Kazuaki Ninomiya $^{\mathrm{a}}$, Ken Inoue ${ }^{\mathrm{b}}$, Yuka Aomori ${ }^{\mathrm{b}}$, Ayaka Ohnishi $^{\mathrm{b}}$, Chiaki Ogino ${ }^{\mathrm{c}}$, Nobuaki Shimizu ${ }^{\mathrm{a}}$, and Kenji Takahashi ${ }^{\mathrm{b}, *}$

${ }^{a}$ Institute of Nature and Environmental Technology,

Kanazawa University, Kakuma-machi, Kanazawa 920-1192, Japan

${ }^{\mathrm{b}}$ Division of Natural System, Graduate School of Natural Science and Technology, Kanazawa University, Kakuma-machi, Kanazawa 920-1192, Japan

${ }^{c}$ Department of Chemical Science and Engineering, Graduate School of Engineering, Kobe University, 1-1 Rokkodaicho, Nada-ku, Kobe 657-8501, Japan

* Corresponding author.

Tel.:+81 76234 4828; fax:+81 762344829

E-mail address: ktkenji@staff.kanazawa-u.ac.jp (K. Takahashi) 


\begin{abstract}
Choline acetate, a totally bio-derived ionic liquid (IL), was used for IL-assisted pretreatment and subsequent fractionation of bagasse into carbohydrate and lignin. The fractionated carbohydrate-rich material (CRM) and lignin-rich material (LRM) were $79.6 \%$ and $5.2 \%$ of the original bagasse, respectively. CRM contained nearly all carbohydrate and approximately $50 \%$ of lignin in the original bagasse. In addition, approximately $20 \%$ of lignin in the original bagasse was fractionated as LRM. The cellulose saccharification percentage was over 95\% for CRM, although the value was only $20 \%$ for the original bagasse. The average molecular weight of LRM was $4.2 \times 10^{4}$, which was approximately ten-times lower than that of the hydrolysis residue of CRM. After five cycles of the processes, the recovery of choline acetate was $80 \%$ of the fresh one. Throughout the five cycles, choline acetate maintained its chemical structure and exhibited its pretreatment capability for fractionation and enzymatic hydrolysis of bagasse.
\end{abstract}

\title{
Keywords:
}

Choline acetate; Ionic liquid; Lignocellulose; Pretreatment; Fractionation; Recycle. 


\section{Introduction}

In the biorefining of lignocellulosic materials into biofuels and bioplastic, the process consists of a pretreatment for enhancing the subsequent enzymatic saccharification of cellulose and hemicellulose, enzymatic hydrolysis of cellulose and hemicellulose to fermentable sugars, and microbial fermentation of these sugars to alcohols and platform chemicals [1]. In particular, pretreatment is an important step because it strongly affects the methodology and productivity of the subsequent hydrolysis and fermentation processes. In this pretreatment step, the hydrogen bonds in crystalline cellulose and covalent cross-linkages in the lignin structure must be disrupted to ensure efficient enzymatic hydrolysis of the lignocellulose [2].

Ionic liquids (ILs), generally defined as organic salts that melt below $100^{\circ} \mathrm{C}$, have been closely studied because they are designable, thermally stable, nonvolatile, and capable of dissolving even polymeric compounds including cellulose under mild conditions [3,4]. Cellulose reprecipitated after being dissolved in ILs could show enhanced enzymatic hydrolysis because of a decrease in its crystallinity [5]. Given that ILs can dissolve not only cellulose but also biomass [6], many researchers have extended this IL-assisted method of cellulose pretreatment to various lignocellulosic biomasses [7-9]. Some groups have demonstrated an IL-assisted fractionation of carbohydrate and lignin from lignocellulosic biomass for enhancing enzymatic hydrolysis of carbohydrate and/or utilizing lignin as feedstock for value-added products [10, 11]. Carbohydrate-enriched lignocellulosic material and pure lignin could be separately recovered as a precipitate and supernatant, respectively, after dissolving the lignocellulosic material in IL and followed by the addition of a suitable antisolvent such as acetone/water $1: 1(\mathrm{v} / \mathrm{v})$ [12] and $0.1 \mathrm{~N} \mathrm{NaOH}$ [13]. However, to the best of our 
knowledge, reports on the fractionation of IL-based biomass to carbohydrate and lignin have been limited to ILs consisting of imidazolium-type IL [11].

Large amounts of ILs are required for the pretreatment of lignocellulosic materials for biorefining on an industrial scale. In general, most ILs currently used for the pretreatment of biomass contain an imidazolium cation; however, imidazolium ILs are expensive. The imidazolium cations have an inhibitory effect on saccharolytic enzymes and fermentative microorganisms used in biorefining [14,15], are not biodegradable [16], and are toxic to organisms in the environment [17]. In contrast, ILs containing ions derived from naturally occurring bases and acids have received attention as "completely bio-derived ILs" that have the potential to solve the problems of conventional imidazolium ILs. The typical examples are ILs that contain cholinium cations combined with amino acid-based anions ([Ch][AA] ILs) [18] or carboxylic acid-based anions ([Ch][CA] ILs) [19]. These cholinium ILs are intrinsically less expensive because of the lower cost of the cation starting material [20]. Furthermore, they are more biocompatible [21] and biodegradable [16] than imidazolium ILs. In a few studies, [Ch][AA] ILs [22,23] and [Ch][CA] ILs [24] have been used for the pretreatment of lignocellulosic materials to enhance enzymatic hydrolysis. However, to the best of our knowledge, there is no report on the IL-assisted pretreatment and fractionation of lignocellulosic biomass using cholinium IL. Nor is there a report on the use of recycled cholinium IL during the pretreatment of lignocellulosic biomass.

In the present study, we performed the IL-assisted pretreatment and subsequent fractionation of lignocellulosic biomass into carbohydrate and lignin using choline acetate (ChOAc) as a completely bio-derived IL. We characterized fractionated carbohydrate-rich materials (CRMs) and lignin-rich materials (LRMs) with respect to 
their chemical structures, enzymatic digestibility, and molecular weight. In addition, we demonstrated the use of recycled ChOAc for the IL-assisted pretreatment and subsequent fractionation of lignocellulosic biomass.

\section{Materials and Methods}

\subsection{Lignocellulosic material and reagents}

Bagasse powder, microcrystalline cellulose, xylan, and alkali lignin were used as the lignocellulosic material, model cellulose, model hemicellulose, and model lignin, respectively. Bagasse was ground and sieved to obtain a powder of $250-500 \mu \mathrm{m}$. Microcrystalline cellulose (Avicel ${ }^{\circledR}$ PH-101), xylan (from beechwood), and alkali lignin (low sulfonate content) were obtained from Sigma-Aldrich (St. Louis, MO, USA). ChOAc was used as a model cholinium IL because it was demonstrated to be nearly as efficient as imidazolium IL in terms of the pretreatment of lignocellulosic materials [24]. ChOAc was prepared using a one-pot neutralization method [25] with minor modifications. In brief, an equimolar amount of acetic acid was dropwise added to a choline hydroxide solution (45 wt\%) in methanol (Sigma-Aldrich) with cooling. The mixture was stirred at room temperature (RT) for $6 \mathrm{~h}$. Water and methanol were removed in vacuo using a rotary evaporator at $40^{\circ} \mathrm{C}$ for $1 \mathrm{~h}$, followed by $90^{\circ} \mathrm{C}$ for $2 \mathrm{~h}$. The resultant residue was dried under vacuum at RT for $16 \mathrm{~h}$. The water content for ChOAc was below 0.5\% wt by Karl-Fischer titration (AQ-2200, Hiranuma Sangyo Co., Ltd, Mito, Japan). Cellulase from Trichoderma viride [Cellulase Onozuka, 3000 filter paper units (FPU) per gram] was purchased by Yakult Pharmaceutical Industry Co., Ltd. (Tokyo, Japan). All other chemicals were from commercial sources and of reagent grade. 


\subsection{IL-assisted biomass fractionation}

\subsubsection{Pretreatment of biomass in IL and subsequent fractionation}

Figure 1 is a schematic diagram of IL-assisted pretreatment and subsequent fractionation of bagasse. The processes include IL-assisted pretreatment of lignocellulosic biomass, fractionation of carbohydrate/lignin, and enzymatic hydrolysis of the fractionated carbohydrate. Bagasse was pretreated with IL and subsequently fractionated into CRM and LRM according to the method reported by Sun et al. [12] with minor modification. Then, CRM was enzymatically hydrolyzed to obtain the lignin-rich hydrolysis residue and the hydrolysate consisting of monomeric sugars.

For the pretreatment step, $0.5 \mathrm{~g}$ of bagasse powder was added to a 30-mL glass vial containing $5 \mathrm{~g}$ of $\mathrm{IL}$. The biomass/IL mixture in a vial was stirred at $110^{\circ} \mathrm{C}$ in a dry oven with magnetic stirring (1,200 rpm) for $16 \mathrm{~h}$. For the fractionation step, the mixture was diluted with $45 \mathrm{~mL}$ of acetone/water (1:1), which resulted in the precipitation of CRM. After stirring, the 50-mL tube containing the suspension was centrifuged $(8,000$ $\times g$ ) for 10 min at $25^{\circ} \mathrm{C}$, and residual CRM was separated from the supernatant. The CRM wash step was repeated five times to remove IL. The recovered CRM was dried in an oven at $90^{\circ} \mathrm{C}$ for $24 \mathrm{~h}$, measured gravimetrically, and ground into a powder using a homogenizer (Fastprep ${ }^{\circledR}$ FP100A, MP Biomedicals LLC., Solon, OH, USA). The acetone was evaporated from the supernatant separated from CRM, which resulted in the precipitation of LRM. The 50-mL tube containing the suspension was centrifuged, washed, dried, and measured as described for CRM.

\subsubsection{Recovery of IL}


The supernatant separated from the LRM preparation was pooled with the waste water generated during the washes of CRM and LRM. From the IL aqueous solution, water was removed in vacuo using a rotary evaporator at $90^{\circ} \mathrm{C}$ for $2 \mathrm{~h}$. The resulting residue was dried under vacuum at RT for $16 \mathrm{~h}$. The recovered IL was measured gravimetrically. The process shown in Fig. 1 was repeated five times by recycling ChOAc. After the second cycle, the recovered IL was used without any supplementation of fresh IL.

\subsubsection{Enzymatic hydrolysis of CRM to obtain hydrolysate and residue}

The enzymatic hydrolysis of CRM was conducted in $30-\mathrm{mL}$ vials at $50^{\circ} \mathrm{C}$ using a rotary shaker at $130 \mathrm{rpm}$. The reaction mixture comprised $0.03 \mathrm{~g}$ of biomass and $5 \mathrm{~mL}$ of a cellulase solution $(62 \mathrm{U} / \mathrm{mL})$ in $50 \mathrm{mM}$ phosphate buffer $(\mathrm{pH}=5.0)$ with $1 \%$ toluene to prevent contamination. The enzymatic reaction was conducted for $48 \mathrm{~h}$, during which samples were collected after 0,24 , and $48 \mathrm{~h}$ and heated at $90^{\circ} \mathrm{C}$ for $5 \mathrm{~min}$ to inactivate the enzyme. After centrifugation of the heated sample at $21,500 \times g$ for 1 min, the glucose and xylose concentrations in the hydrolysate was determined by high-performance liquid chromatography (HPLC), as describe below. Cellulose saccharification was evaluated as the percentage of cellulose hydrolyzed into glucose to cellulose in the bagasse recovered from the pretreatment. After enzymatic reaction for $48 \mathrm{~h}$, the solid residue was corrected by centrifugation at $8,000 \times g$ for $10 \mathrm{~min}$ at $25^{\circ} \mathrm{C}$. The washing of the residue was repeated several times, and the recovered residue was dried in an oven at $90^{\circ} \mathrm{C}$ for $24 \mathrm{~h}$ and measured gravimetrically.

\subsection{Analytical methods}




\subsubsection{Compositional analyses of biomass}

The cellulose, hemicellulose, and lignin contents of the original bagasse, CRM, and LRM were determined using TAPPI methods [26,27] with minor modifications. In brief, $0.1 \mathrm{~g}$ of sample was mixed with $2 \mathrm{~mL}$ of a $72 \%(v / v) \mathrm{H}_{2} \mathrm{SO}_{4}$ aqueous solution for $2 \mathrm{~h}$ at RT. The mixture was transferred to a 200-mL Erlenmeyer flask, diluted with 75 $\mathrm{mL}$ of water, and autoclaved at $121^{\circ} \mathrm{C}$ for $15 \mathrm{~min}$. The acid-diluted hydrolysate was filtered, following which the amount of acid-insoluble lignin was gravimetrically determined by measuring the residue on the filter after drying at $100^{\circ} \mathrm{C}$ for $12 \mathrm{~h}$. The amount of acid-soluble lignin was determined from the ultraviolet (UV) absorbance of the filtrate at $205 \mathrm{~nm}$ and the absorption coefficient of $110 \mathrm{~L} \mathrm{~g}^{-1} \mathrm{~cm}^{-1}$ [27]. The sum of the acid-insoluble and acid-soluble lignin was regarded to be the total lignin amount. The amount of sugars in the hydrolysate was determined by HPLC equipped with a refractive index (RI) detector (Shimadzu Co., Kyoto, Japan) using a CARBOSep CHO-682 column (Tokyo Chemical Industry Co., Ltd, Tokyo, Japan). The column was run at $85^{\circ} \mathrm{C}$ with a water mobile phase and a flow rate of $0.4 \mathrm{~mL} / \mathrm{min}$. The amount of cellulose and hemicellulose was calculated from the glucose and xylose content multiplied by anhydro correction factors of 162/180 and 132/150, respectively.

\subsubsection{Fourier-transform infrared (FTIR) spectrometry of biomass and IL}

For FTIR analysis of biomass, $10 \mathrm{mg}$ of the solid sample was mixed with $50 \mathrm{mg}$ of spectroscopic-grade $\mathrm{KBr}$, ground, and pressed to produce transparent pellets using a laboratory mill and press (QM-1 and QP-1, Chromato Science, Osaka, Japan). Analyses were conducted using an FTIR spectrometer (Nexus 470, Thermo Fisher Scientific K.K., Yokohama, Japan) over the 4,000-400 $\mathrm{cm}^{-1}$ range with a resolution of $2 \mathrm{~cm}^{-1}$ and 32 
scans per sample. The background spectrum of pure $\mathrm{KBr}$ was subtracted from the spectrum of the sample. For cellulosic material, the total crystallinity index (TCI) [28] was calculated as $A_{1372} / A_{2894}$, where $A_{1372} / A_{2894}$ denotes the ratio of peak areas at 1372 $\mathrm{cm}^{-1}$ and $2894 \mathrm{~cm}^{-1}$ obtained from the FTIR spectrum. For lignin material, the peak area at a given peak was normalized by the peak area at $1595 \mathrm{~cm}^{-1}\left(A_{X} / A_{1595}\right)$, where the peak at $1595 \mathrm{~cm}^{-1}$ corresponds to the aromatic skeletal C=C stretching vibration [29].

\subsubsection{Gel permeation chromatography (GPC) analysis of biomass}

For GPC analysis of lignin, $10 \mathrm{mg}$ of the lignin sample was dissolved in $1 \mathrm{~mL}$ of $N, N$-dimethylformamide (DMF) at RT for $1 \mathrm{~h}$. The lignin-DMF mixture was then filtered, and the DMF-soluble lignin fraction was used for analysis. The amount of DMF-insoluble lignin was gravimetrically determined by measuring the residue on the filter after drying at $100^{\circ} \mathrm{C}$ for $12 \mathrm{~h}$. The molecular weight of the lignin sample was determined by GPC equipped with an RI detector (Shimadzu Co., Kyoto, Japan) using a TSKgel $\alpha-\mathrm{M}$ column (Tosoh Corporation, Tokyo, Japan). The operating conditions were as follows: $40^{\circ} \mathrm{C}$ with a mobile phase of DMF containing $10 \mathrm{mM}$ lithium bromide at a flow rate of $1.0 \mathrm{~mL} / \mathrm{min}$. For the calibration, monodisperse polystylene of known molecular weight was used as the standard. Average molecular weight $\left(M_{\mathrm{w}}\right)$, number-average molecular weight $\left(M_{\mathrm{n}}\right)$, and polydispersity $\left(M_{\mathrm{w}} / M_{\mathrm{n}}\right)$ were determined from the chromatogram.

\subsubsection{Nuclear magnetic resonance (NMR) analysis of IL}

For NMR analysis of IL, $30 \mathrm{mg}$ of IL was dissolved in $500 \mu \mathrm{L}$ of DMSO-d $\mathrm{d}_{6}{ }^{1} \mathrm{H}-$ and ${ }^{13} \mathrm{C}$-NMR spectra of the sample were recorded at $25^{\circ} \mathrm{C}$ using a $600 \mathrm{MHz} \mathrm{NMR}$ 
spectrometer (JNM-ECA600, JEOL Ltd., Tokyo, Japan). For each sample, 32 and 256 scans were accumulated for ${ }^{1} \mathrm{H}$ - and ${ }^{13} \mathrm{C}$-NMR spectra, respectively.

\section{Results}

\subsection{Mass and composition of biomass materials fractionated from bagasse}

In the present study, totally bio-derived IL ChOAc was used for IL-assisted pretreatment and subsequent fractionation of bagasse into CRM and LRM. As listed in Table 1, relative masses of fractionated CRM and LRM were $79.6 \%$ and $5.2 \%$, respectively, of the original bagasse. In addition, $15.2 \%$ of the original bagasse could not be recovered in this process, which was considered to remain dissolved in ChOAc recovered from this process. According to compositional analyses of the fractionated CRM and LRM, it was found that almost all the carbohydrate with approximately 50\% of lignin in the original bagasse was recovered as CRM and approximately $20 \%$ of the lignin in the original bagasse was fractionated as LRM.

To confirm the fractionation results shown in Table 1, FTIR analyses were conducted. Figure 2 shows the FTIR spectra of the original bagasse and the fractionated CRM and LRM. Some peaks correlating to cellulose, hemicellulose, and lignin [29-31] were observed upon analysis of the original bagasse (Fig. 2A). Characteristic peaks associated with carbohydrate (cellulose and hemicellulose) were observed in the fractionated CRM (Fig. 2B) as well as authentic samples [microcrystalline cellulose (Fig. 2D) and xylan (Fig. 2E)] at $1372 \mathrm{~cm}^{-1}$ (C-H bending vibration), $1163 \mathrm{~cm}^{-1}$ (C-O-C asymmetric stretching vibration), $1100 \mathrm{~cm}^{-1}$ (O-H association band), 1060 $\mathrm{cm}^{-1}$ (C-O stretching vibration), and $896 \mathrm{~cm}^{-1}$ (C-H bending vibration). In contrast, characteristic peaks associated with lignin were observed in the fractionated LRM (Fig. 
2F) as well as authentic alkali lignin (Fig. 2J) at $1595 \mathrm{~cm}^{-1}$ (aromatic skeletal $\mathrm{C}=\mathrm{C}$ stretching vibration), $1512 \mathrm{~cm}^{-1}$ (aromatic skeletal C-C stretching vibration), $1456 \mathrm{~cm}^{-1}$ (asymmetric bending vibration of $\mathrm{CH}_{3}$ in lignin), $1424 \mathrm{~cm}^{-1}$ (aromatic $\mathrm{C}-\mathrm{H}$ in-plane bending vibration), $1328 \mathrm{~cm}^{-1}$ (syringyl unit breathing with $\mathrm{C}=\mathrm{O}$ stretching vibration and condensed guaiacyl unit), $1271 \mathrm{~cm}^{-1}$ (guaiacyl unit breathing with $\mathrm{C}=\mathrm{O}$ stretching vibration), $1125 \mathrm{~cm}^{-1}$ (ether-O-), $1032 \mathrm{~cm}^{-1}$ [aromatic C-H in-plane bending vibration, $\mathrm{C}-\mathrm{O}$ bending vibration in primary alcohols, and $\mathrm{C}=\mathrm{O}$ stretch vibration (unconjugated)],

and $830 \mathrm{~cm}^{-1}$ (aromatic C-H out-of-plane bending vibration). These characteristic peaks associated with lignin are not significant in the fractionated CRM and vice versa. Overall, these FTIR data qualitatively support the results of bagasse fractionation assisted by IL pretreatment.

\subsection{Characterization of CRM}

To compare the original bagasse powder and fractionated CRM in terms of the enzymatic saccharification efficiency, enzymatic hydrolysis of the biomass sample was conducted for $48 \mathrm{~h}$. As shown in Table 2, the cellulose saccharification percentages were approximately $20 \%$ and $40 \%$ for the original bagasse and microcrystalline cellulose, respectively. In contrast, the cellulose saccharification percentage reached over 95\% in the case of CRM. These results indicate that CRM obtained by IL-assisted pretreatment and fractionation enables higher accessibility of the cellulase enzyme to the cellulose substrate.

To examine the crystalline structures of the original bagasse powder and the fractionated CRM, TCI $\left(A_{1372} / A_{2894}\right)$ was determined as the quantitative index of the overall crystallinity of cellulose on the basis of FTIR spectra shown in Fig. 2. Higher 
TCI values indicate that the materials have a higher crystallinity and more ordered cellular structure [28]. As listed in Table 2, TCI values for the original bagasse and microcrystalline cellulose were 0.085 and 0.123 , respectively. In contrast, the value decreased to 0.039 for CRM. Thus, we confirmed that IL-assisted biomass pretreatment and fractionation could mitigate the crystalline and ordered structure of cellulose in the bagasse, resulting in higher cellulose saccharification efficiency.

\subsection{Characterization of LRM and hydrolysis residue of CRM}

In the present study, the fractionated CRM was enzymatically hydrolyzed to obtain the lignin-rich residue, as illustrated in Fig. 1. According to compositional analysis listed in Table 1, under the examined conditions, cellulose and hemicellulose saccharification percentages were $95 \%$ and approximately $70 \%$, respectively. This indicates that the hydrolysis residue of CRM obtained in the present study was lignin rich but included a considerable amount of carbohydrate.

To compare the molecular weights of LRM and hydrolysis residue of CRM, GPC analyses were conducted. Prior to GPC analysis, the sample was mixed in DMF at a concentration of $1 \%(\mathrm{w} / \mathrm{v})$. LRM and authentic alkali lignin were completely dissolved in DMF, while the solubility of the hydrolysis residue of CRM in DMF was only 24\%. This result indicates that compared with the fractionated LRM, the hydrolysis residue of CRM has a highly macromolecular structure. Figure 3 shows the GPC chromatogram for the lignin sample solubilized in DMF. The chromatograms for LRM and alkali lignin overlapped with each other, while that for the hydrolysis residue of CRM displayed a larger molecular weight. As listed in Table 3, the $M_{\mathrm{w}}$ values were $4.2 \times 10^{4} \mathrm{~g} / \mathrm{mol}$ and $3.8 \times 10^{5} \mathrm{~g} / \mathrm{mol}$ for LRM and the hydrolysis residue of CRM, respectively. The $M_{\mathrm{n}}$ 
values were $5.2 \times 10^{3} \mathrm{~g} / \mathrm{mol}$ and $4.6 \times 10^{4} \mathrm{~g} / \mathrm{mol}$ for LRM and the hydrolysis residue of CRM, respectively. The $M_{\mathrm{w}} / M_{\mathrm{n}}$ values were 8.1 and 8.3 for LRM and the hydrolysis residue of CRM, respectively. These results revealed that the molecular weight of LRM was approximately ten-times lower than that of the hydrolysis residue of CRM.

To examine the chemical structures of LRM and the hydrolysis residue of CRM, FTIR analysis was conducted. The FTIR spectrum for the hydrolysis residue of CRM (Fig. 2H) was almost the same as that for LRM (Fig. 2F), except that the peaks at 1125 $\mathrm{cm}^{-1}$ and $1032 \mathrm{~cm}^{-1}$ for CRM were broader than those for LRM. This result is consistent with compositional analysis of the hydrolysis residue of CRM (Table 1), which showed that some hemicellulose remains in the hydrolysis residue of CRM. Therefore, the lignin peaks at $1125 \mathrm{~cm}^{-1}$ and $1032 \mathrm{~cm}^{-1}$ overlapped with the peaks derived from carbohydrate. Moreover, the $A_{1700} / A_{1595}$ value and the $A_{1375} / A_{1595}$ value were determined as quantitative indices of carbonyl group and phenolic $\mathrm{OH}$ group, respectively, from the FTIR spectra shown in Fig. 2. The lignin macromolecule with a higher amount of carbonyl and phenolic groups can be useful when higher reactivity is required [29]. As listed in Table 3, the $A_{1700} / A_{1595}$ values were 0.415 and 0.421 for LRM and the hydrolysis residue of CRM, respectively, which were higher than the value for alkali lignin. In contrast, the $A_{1375} / A_{1595}$ values were 0.031 and 0.082 for LRM and the hydrolysis residue of CRM, respectively, which were lower than the value for alkali lignin. Overall, it was found that LRM obtained by IL-assisted biomass pretreatment and fractionation had the same molecular weight and a higher amount of the carbonyl group than conventional alkali lignin.

\subsection{Characterization of recycled ChOAc}


In the present study, IL-assisted biomass pretreatment and subsequent fractionation (Fig. 1) were repeated five times by recycling ChOAc. First, the recovery of ChOAc was evaluated in the respective cycles of process. As listed in Table 4, the recovery percentage of ChOAc used for each cycle was approximately $90 \%-100 \%$. At the completion of the five cycles, the overall recovery percentage became approximately $80 \%$ of the fresh ChOAc applied for the first cycle.

To examine the changes in the chemical structure of ChOAc recovered during the five cycles of the process described above, its ${ }^{1} \mathrm{H}$ - and ${ }^{13} \mathrm{C}$-NMR spectra were monitored. As shown in Figs. S1 and S2, there was no significant difference in the NMR spectra between fresh and recycled ChOAc, indicating that its chemical structure was maintained throughout the processes under the examined condition.

The results of bagasse fractionation in the fifth cycle of the process were approximately the same as those in the first cycle, in which fresh ChOAc was used (data not shown). The FTIR spectra of the fractionated CRM and LRM in the fifth cycle (Fig. 2C and $\mathrm{G}$ ) were almost same as those in the first cycle using fresh ChOAc (Fig. 2B and F). Moreover, as listed in Table 2, the cellulose saccharification percentage and the TCI value in the fifth cycle were at the same as those in the first cycle. Furthermore, as listed in Table 3, the average molecular weight and indices of the carbonyl group and phenolic $\mathrm{OH}$ group in the fifth cycle were at the same level as those in the first cycle. Overall, totally bio-derived IL ChOAc maintained its chemical structure and pretreatment capability of lignocellulosic biomass even in five cycles of the processes under the conditions examined in the present study.

\section{Discussion}


Till date, some studies have demonstrated the IL-assisted fractionation of carbohydrate and lignin from lignocellulosic biomass by employing antisolvents, such as acetone/water $1: 1(\mathrm{v} / \mathrm{v})$ [12] and $\mathrm{NaOH}$ solution [13], for cellulosic material and solvents for lignin. An alkali-free method using acetone/water [12] is better because all solvents used for the fractionation of biomass (IL and acetone) can be recovered without generating waste water by neutralization. However, when acetone/water is used, the lignin fractionation efficiency is still low (approximately 30\% of lignin is recovered from the original biomass). In comparison, our results demonstrate that employing completely bio-derived IL ChOAc during the fractionation of lignin yielded approximately $20 \%$ of the lignin from the original biomass (Table 1). Holocellulose (cellulose and hemicellulose) and lignin bind to each other, forming a lignin-carbohydrate complex (LCC) that prevents the efficient fractionation of biomass components. To enhance the fractionation efficiency, we employed ultrasound irradiation for ChOAc-assisted pretreatment prior to fractionation instead of conventional heating. However, there was no marked enhancement in the fractionation efficiency (data not shown). Recently, it has been reported [32] that using acidic IL (i.e., 1-H-3-methylimidazolium chloride) for the pretreatment step and acetone/water for the fractionation step yielded a higher fractionation efficiency. However, this method caused both hemicellulose and lignin to depolymerize and dissolve in ILs during pretreatment [32]. Taken together, these results suggest that further research is necessary to develop an IL-assisted fractionation method with a clear separation of carbohydrate and lignin.

Cellulose crystallinity is a major barrier to the enzymatic hydrolysis of lignocellulosic/cellulosic materials because a rigid crystalline structure prevents the 
enzymes to access polysaccharides. In addition, lignin is an obstacle to the enzymatic hydrolysis of lignocellulosic materials because a highly branched network structure with covalent cross-linkages to hemicellulose also prevents the enzymes to access polysaccharides. In the present study, ChOAc-assisted pretreatment followed by the fractionation of lignin enhanced saccharification efficiency (Table 1). However, in our previous study [24], the cellulose saccharification ratio reached 95\% even after the ChOAc-assisted pretreatment of lignocellulosic biomass without a lignin fractionation step. In that study, the lignin content in the pretreated biomass was almost the same as in the untreated original material. This could be because the crystalline cellulose structure, rather than the lignin network structure, was the principal barrier for enzyme accessibility. We also hypothesized that not removal but mitigation of the lignin structure was sufficient to allow the enzyme molecules to access the polysaccharides. Therefore, the removal of lignin is not necessary for enhancing the efficiency of enzymatic hydrolysis. Nonetheless, the fractionation of lignin is important for utilizing lignin as feedstock for value-added products.

For the use of lignin as a raw material of resin, epoxy resin, and phenol resin have been intensively studied; in particular, epoxy resin prepared from either kraft lignin, a byproduct of the pulping industry, or lignin that has been fractionated via the pretreatment biomass processes such as steam explosion [33]. However, to the best of our knowledge, there are no reports of epoxy resin using lignin that has been fractionated from IL-pretreated lignocellulosic biomass. At present, we are studying epoxy resin using lignin fractionated after an IL-assisted pretreatment. In addition, we are investigating whether its properties are comparable with those of the conventional lignin-epoxy resin prepared from industrial kraft lignin. 
With respect to the use of recycled IL, the evaporation of water has generally been employed to recover IL from diluted aqueous solution. This solution is generated by a washing procedure during the pretreatment and fractionation process. The method is simple but requires energy for large-scale application. Moreover, as observed in the present study, the depolymerized lignin and other low-molecular-weight compounds were still dissolved in IL that was recovered by the evaporation of water. This results in a loss of biomass that could be utilized and may cause a loss of pretreatment capability following repeated use. Recently, it was reported that IL could be separated and concentrated from the diluted aqueous solution by ion-exclusion chromatography [34,35] or electrodialysis with an ion-exchange membrane [36-38]. These separation principles are useful when IL is recovered from diluted aqueous solution containing depolymerized lignin monomer [37] or monosaccharide [34,35,38], which is likely to be generated during the IL-assisted pretreatment of lignocellulosic biomass or one-pot IL-assisted pretreatment and hydrolysis of (ligno)cellulosic biomass, respectively. Therefore, for the large-scale application of ILs in the biomass pretreatment process, the development of simple, energy-saving, and efficient IL recovery methods is required.

\section{Conclusions}

Totally bio-derived ChOAc was used for IL-assisted pretreatment and fractionation of bagasse into CRM and LRM. The fractionated CRM mostly contained carbohydrate and approximately 50\% of lignin from the bagasse. LRM was almost pure lignin. In contrast to the original bagasse, CRM was enzymatically hydrolyzed to glucose and xylose. The average molecular weight of LRM was approximately ten-times lower than that of the hydrolysis residue of CRM. After the five cycles of the processes, the 
recovery of ChOAc was $80 \%$ of the fresh one; it maintained its chemical structure and exhibited its pretreatment capability for the fractionation and enzymatic hydrolysis of bagasse.

\section{Acknowledgments}

This study was supported in part by an Advanced Low Carbon Technology Research and Development Program (ALCA) (Grant number 2100040) of the Japan Science and Technology Agency of Japan. This study was supported in part by a Center of Innovation Science and Technology based Radical Innovation and Entrepreneurship Program (COIstream) of the Japan Science and Technology Agency of Japan.

\section{References}

[1] M.G. Adsul, M.S. Singhvi, S.A. Gaikaiwari, D.V. Gokhale, Development of biocatalysts for production of commodity chemicals from lignocellulosic biomass, Bioresour. Technol. 102 (2011) 4304-4312.

[2] P. Alvira, E. Tomás-Pejó, M. Ballesteros, M.J. Negro, Pretreatment technologies for an efficient bioethanol production process based on enzymatic hydrolysis: A review, Bioresour. Technol. 101 (2010) 4851-4861.

[3] H. Olivier-Bourbigou, L. Magna, D. Morvan, Ionic liquids and catalysis: Recent progress from knowledge to applications, Appl. Catal., A 373 (2010) 1-56.

[4] R.P. Swatloski, S.K. Spear, J.D. Holbrey, R.D. Rogers, Dissolution of cellose with ionic liquids. J. Am. Chem. Soc. 124 (2002) 4974-4975.

[5] A.P. Dadi, S. Varanasi, C.A. Schall, Enhancement of cellulose saccharification kinetics using an ionic liquid pretreatment step, Biotechnol. Bioeng. 95 (2006) 
904-910.

[6] D.A. Fort, R.C. Remsing, R.P. Swatloski, P. Moyna, G. Moyna, R.D. Rogers, Can ionic liquids dissolve wood? Processing and analysis of lignocellulosic materials with 1-n-butyl-3-methylimidazolium chloride, Green Chem. 9 (2007) 63-69.

[7] I. Kilpeläinen, H. Xie, A. King, M. Granstrom, S. Heikkinen, D.S. Argyropoulos, Dissolution of wood in ionic liquids, J. Agric. Food Chem. 55 (2007) 9142-9148.

[8] S.H. Lee, T.V. Doherty, R.J. Linhardt, J.S. Dordick, Ionic liquid-mediated selective extraction of lignin from wood leading to enhanced enzymatic cellulose hydrolysis, Biotechnol. Bioeng. 102 (2009) 1368-1376.

[9] Q. Li, Y.-C. He, M. Xian, G. Jun, X. Xu, J.-M. Yang, L.Z. Li, Improving enzymatic hydrolysis of wheat straw using ionic liquid 1-ethyl-3-methyl imidazolium diethyl phosphate pretreatment, Bioresour. Technol. 100 (2009) 3570-3575.

[10] N. Sun, H. Rodríguez, M. Rahman, R.D. Rogers, Where are ionic liquid strategies most suited in the pursuit of chemicals and energy from lignocellulosic biomass?, Chem. Commun. 47 (2010) 1405-1421.

[11] A. Brandt, J. Gräsvik, J.P. Hallett, T. Welton, Deconstruction of lignocellulosic biomass with ionic liquids, Green Chem. 15 (2013) 550.

[12] N. Sun, M. Rahman, Y. Qin, M.L. Maxim, H. Rodríguez, R.D. Rogers, Complete dissolution and partial delignification of wood in the ionic liquid 1-ethyl-3-methylimidazolium acetate, Green Chem. 11 (2009) 646-655.

[13] S.S.Y. Tan, D.R. MacFarlane, J. Upfal, L.A. Edye, W.O.S. Doherty, A.F. Patti, J.M. Pringle, J.L. Scott, Extraction of lignin from lignocellulose at atmospheric pressure using alkylbenzenesulfonate ionic liquid, Green Chem. 11 (2009) 
339-345.

[14] S. Datta, B. Holmes, J.I. Park, Z. Chen, D.C. Dibble, M. Hadi, H.W. Blanch, B.A. Simmons, R. Sapra, Ionic liquid tolerant hyperthermophilic cellulases for biomass pretreatment and hydrolysis, Green Chem. 12 (2010) 338-345.

[15] M. Ouellet, S. Datta, D.C. Dibble, P.R. Tamrakar, P.I. Benke, C. Li, S. Singh, K.L. Sale, P.D. Adams, J.D. Keasling, B.A. Simmons, B.M. Holmes, A. Mukhopadhyay, Impact of ionic liquid pretreated plant biomass on Saccharomyces cerevisiae growth and biofuel production, Green Chem. 13 (2011) 2743-2749.

[16] R. Boething, E. Sommer, D. DiFiore, Designing small molecules for biodegradability, Chem. Rev. 107 (2007) 2207-2227.

[17] R.F. Frade, C.A. Afonso, Impact of ionic liquids in environment and humans: an overview, Hum. Exp. Toxicol. 29 (2010) 1038-1054.

[18] S. Hu, T. Jiang, Z. Zhang, A. Zhu, B. Han, J. Song, Y. Xie, W. Li, Functional ionic liquid from biorenewable materials: synthesis and application as a catalyst in direct aldol reactions, Tetrahedron Lett. 48 (2007) 5613-5617.

[19] Y. Fukaya, Y. Iizuka, K. Sekikawa, H. Ohno, Bio ionic liquids: room temperature ionic liquids composed wholly of biomaterials, Green Chem. 9 (2007) $1155-1157$.

[20] N.V. Plechkova, K.R. Seddon, Applications of ionic liquids in the chemical industry, Chem. Soc. Rev. 37 (2008) 123-150.

[21] M. Petkovic, J. Ferguson, A. Bohn, J. Trindade, I. Martins, M.B. Carvalho, M.C. Leitão, C. Rodrigues, H. Garcia, R. Ferreira, K.R. Seddon, L.P.N. Rebelo, C. Silva Pereira, Exploring fungal activity in the presence of ionic liquids, Green 
Chem. 11, (2009) 889-894.

[22] Q.-P. Liu, X.-D. Hou, N. Li, M.-H. Zong, Ionic liquids from renewable biomaterials: synthesis, characterization and application in the pretreatment of biomass, Green Chem. 14 (2012) 304-307.

[23] X.-D. Hou, T.J. Smith, N. Li, M.-H. Zong, Novel renewable ionic liquids as highly effective solvents for pretreatment of rice straw biomass by selective removal of lignin, Biotechnol. Bioeng. (2012) DOI: 10.1002/bit.24522

[24] K. Ninomiya, T. Yamauchi, M. Kobayashi, O. Chiaki, N. Shimizu, K. Takahashi, Cholinium carboxylate ionic liquids for pretreatment of lignocellulosic materials to enhance subsequent enzymatic saccharification. Biochem Eng. J. 71, (2013) 25-29.

[25] Y. Yu, X. Lu, Q. Zhou, K. Dong, H. Yao, S. Zhang, Biodegradable naphthenic acid ionic liquids: synthesis, characterization, and quantitative structure-biodegradation relationship, Chem. Eur. J. 14 (2008) 11174-11782.

[26] TAPPI useful method UM-250, 1991. Acid-soluble lignin in wood and pulp.

[27] TAPPI method T 222 cm-02, 2002. Acid-insoluble lignin in wood and pulp.

[28] M.L. Nelson, R.T. O’Connor, Relation of certain infrared bands to cellulose crystallinity and crystal lattice type. Part II. A new infrared ratio for estimation of crystallinity in celluloses I and II, J. Appl. Polym. Sci. 8 (1964) 1325-1341.

[29] A. Casas, M.V. Alonso, M. Oliet, E. Rojo, F. Rodríguez, FTIR analysis of lignin regenerated from Pinus radiata and Eucalyptus globulus woods dissolved in imidazolium-based ionic liquids, J. Chem. Technol. Biotechnol. 87 (2012) $472-480$.

[30] N. Labbé, T.G. Rials, S.S. Kelley, Z.-M. Cheng, J.-Y. Kim, Y. Li, FT-IR imaging 
and pyrolysis-molecular beam mass spectrometry: new tools to investigate wood tissues, Wood Sci. Technol. 39 (2005) 61-77.

[31] K. Bilba, A. Ouensanga, Fourier transform infrared spectroscopic study of thermal degradation of sugar cane bagasse, J. Anal. Appl. Pyrolysis. 38 (1996) $61-73$.

[32] B.J. Cox, J.G. Ekerdt, Pretreatment of yellow pine in an acidic ionic liquid: Extraction of hemicellulose and lignin to facilitate enzymatic digestion, Bioresour. Technol. 134 (2013) 59-65.

[33] T. Koike, Progress in development of epoxy resin systems based on wood biomass in Japan, Polym. Eng. Sci. 52 (2012) 701-717.

[34] D. Feng, L. Li, F. Yang, W. Tan, G. Zhao, H. Zou, et al., Separation of ionic liquid [Mmim][DMP] and glucose from enzymatic hydrolysis mixture of cellulose using alumina column chromatography., Appl. Microbiol. Biotechnol. 91 (2011) 399-405.

[35] N.L. Mai, N.T. Nguyen, J.-I. Kim, H.-M. Park, S.-K. Lee, Y.-M. Koo, Recovery of ionic liquid and sugars from hydrolyzed biomass using ion exclusion simulated moving bed chromatography., J. Chromatogr. A. 1227 (2012) 67-72.

[36] X. Wang, Y. Nie, X. Zhang, S. Zhang, J. Li, Recovery of ionic liquids from dilute aqueous solutions by electrodialysis, Desalination. 285 (2012) 205-212.

[37] L.T.P. Trinh, Y.J. Lee, J.-W. Lee, H.-J. Bae, H.-J. Lee, Recovery of an ionic liquid [BMIM]Cl from a hydrolysate of lignocellulosic biomass using electrodialysis, Sep. Purif. Technol. 120 (2013) 86-91.

[38] C. Abels, K. Thimm, H. Wulfhorst, A.C. Spiess, M. Wessling, Membrane-based recovery of glucose from enzymatic hydrolysis of ionic liquid pretreated 
cellulose., Bioresour. Technol. 149 (2013) 58-64. 


\section{Figure captions}

Fig. 1 Schematic diagram of IL-assisted pretreatment and subsequent fractionation of bagasse.

Fig. 2 FTIR spectra of cellulosic and lignin materials fractionated from bagasse. (A) original bagasse, (B) CRM from 1st cycle, (C) CRM from 5th cycle, (D) microcrystalline cellulose, (E) xylan, (F) LRM from 1st cycle, (G) LRM from 5th cycle, (H) hydrolysis residue of CRM from 1st cycle, (I) hydrolysis residue of CRM from 5th cycle, and (J) alkali lignin. Vertical lines denote the characteristic peaks of cellulose/hemicellulose (blue lines) and lignin (red lines).

Fig. 3 GPC chromatograms of lignin materials fractionated from bagasse. Red lines: LRM from 1st cycle and 5th cycles. Blue lines: hydrolysis residue of CRM from 1st cycle and 5th cycles. Green lines: alkali lignin. 
Table 1 Mass and composition of cellulosic and lignin materials fractionated from bagasse

\begin{tabular}{|c|c|c|c|c|c|}
\hline \multirow{2}{*}{ Fraction } & \multirow{2}{*}{$\begin{array}{l}\text { Relative mass } \\
\text { [\% of original baggase] }\end{array}$} & \multicolumn{4}{|c|}{$\begin{array}{l}\text { Composition } \\
\text { [\% of original baggase] }\end{array}$} \\
\hline & & Cellulose & Hemicellulose & Lignin & Others \\
\hline Original bagasse & 100 & 37.4 & 29.6 & 25.4 & 7.6 \\
\hline CRM & 79.6 & 36.2 & 25.6 & 13.6 & 4.2 \\
\hline LRM & 5.2 & 0 & 0 & 5.2 & 0 \\
\hline Loss & 15.2 & 1.2 & 4.0 & 6.6 & 3.4 \\
\hline $\begin{array}{l}\text { Hydrolysate } \\
\text { of CRM }\end{array}$ & 58.7 & 34.4 & 17.8 & 2.3 & 4.2 \\
\hline $\begin{array}{l}\text { Hydrolysis residue } \\
\text { of CRM }\end{array}$ & 20.9 & 1.8 & 7.8 & 11.3 & 0 \\
\hline
\end{tabular}


Table 2 Characterization of cellulosic materials fractionated from bagasse.

\begin{tabular}{lll}
\hline Fraction & $\begin{array}{l}\text { Cellulose saccharification } \\
{[\%]}\end{array}$ & $\begin{array}{l}\text { TCI } \\
{[-]}\end{array}$ \\
\hline Original bagasse & $17.5 \pm 1.7$ & 0.085 \\
CRM for 1st cycle & $95.8 \pm 6.6$ & 0.039 \\
CRM for 5th cycle & $94.9 \pm 7.6$ & 0.046 \\
Microcrystalline cellulose & $41.0 \pm 3.2$ & 0.123 \\
\hline
\end{tabular}

Cellulose saccharification ratio is expressed as mean \pm standard error from three independent experiments. TCI $\left(A_{1372} / A_{2894}\right)$ were determined from FTIR spectra. 
Table 3 Characterization of lignin materials fractionated from bagasse.

\begin{tabular}{|c|c|c|c|c|c|}
\hline Fraction & $\begin{array}{l}A_{1700} / A_{1595} \\
{[-]}\end{array}$ & $\begin{array}{l}A_{1375} / A_{1595} \\
{[-]}\end{array}$ & $\begin{array}{l}M_{\mathrm{w}} \\
{[\mathrm{g} / \mathrm{mol}]}\end{array}$ & $\begin{array}{l}M_{\mathrm{n}} \\
{[\mathrm{g} / \mathrm{mol}]}\end{array}$ & $\begin{array}{l}M_{\mathrm{w}} / M_{\mathrm{n}} \\
{[-]}\end{array}$ \\
\hline LRM for 1st cycle & 0.415 & 0.031 & $4.22 \times 10^{4}$ & $5.20 \times 10^{3}$ & 8.12 \\
\hline LRM for 5th cycle & 0.424 & 0.021 & $4.24 \times 10^{4}$ & $5.59 \times 10^{3}$ & 7.58 \\
\hline $\begin{array}{l}\text { Hydrolysis residue of } \\
\text { CRM for 1st cycle }\end{array}$ & 0.421 & 0.082 & $3.84 \times 10^{5}$ & $4.61 \times 10^{4}$ & 8.32 \\
\hline $\begin{array}{l}\text { Hydrolysis residue of } \\
\text { CRM for } 5 \text { th cycle }\end{array}$ & 0.464 & 0.091 & $3.77 \times 10^{5}$ & $4.58 \times 10^{4}$ & 8.23 \\
\hline Alkali lignin & 0.127 & 0.113 & $7.36 \times 10^{4}$ & $5.94 \times 10^{3}$ & 12.39 \\
\hline
\end{tabular}

The $A_{1700} / A_{1595}$ and $A_{1375} / A_{1595}$ values were and determined from FTIR analysis, as indices for carbonyl group and phenolic OH group, respectively. $M_{\mathrm{w}}, M_{\mathrm{n}}$, and $M_{\mathrm{w}} / M_{\mathrm{n}}$ values were determined from GPC analyses. 
Table 4 Recovery of choline acetate from the pretreatment process

\begin{tabular}{lll}
\hline \multirow{2}{*}{$\begin{array}{l}\text { Cycle of } \\
\text { pretreatment process }\end{array}$} & \multicolumn{2}{l}{ Recovery percentage } \\
\cline { 2 - 3 } [\% of IL used for each cycle] & [\% of IL used for 1st cycle] \\
\hline 1st & 92.2 & 92.2 \\
2nd & 99.9 & 92.1 \\
3rd & 89.2 & 82.2 \\
4th & 100.6 & 82.7 \\
5th & 96.3 & 79.6 \\
\hline
\end{tabular}




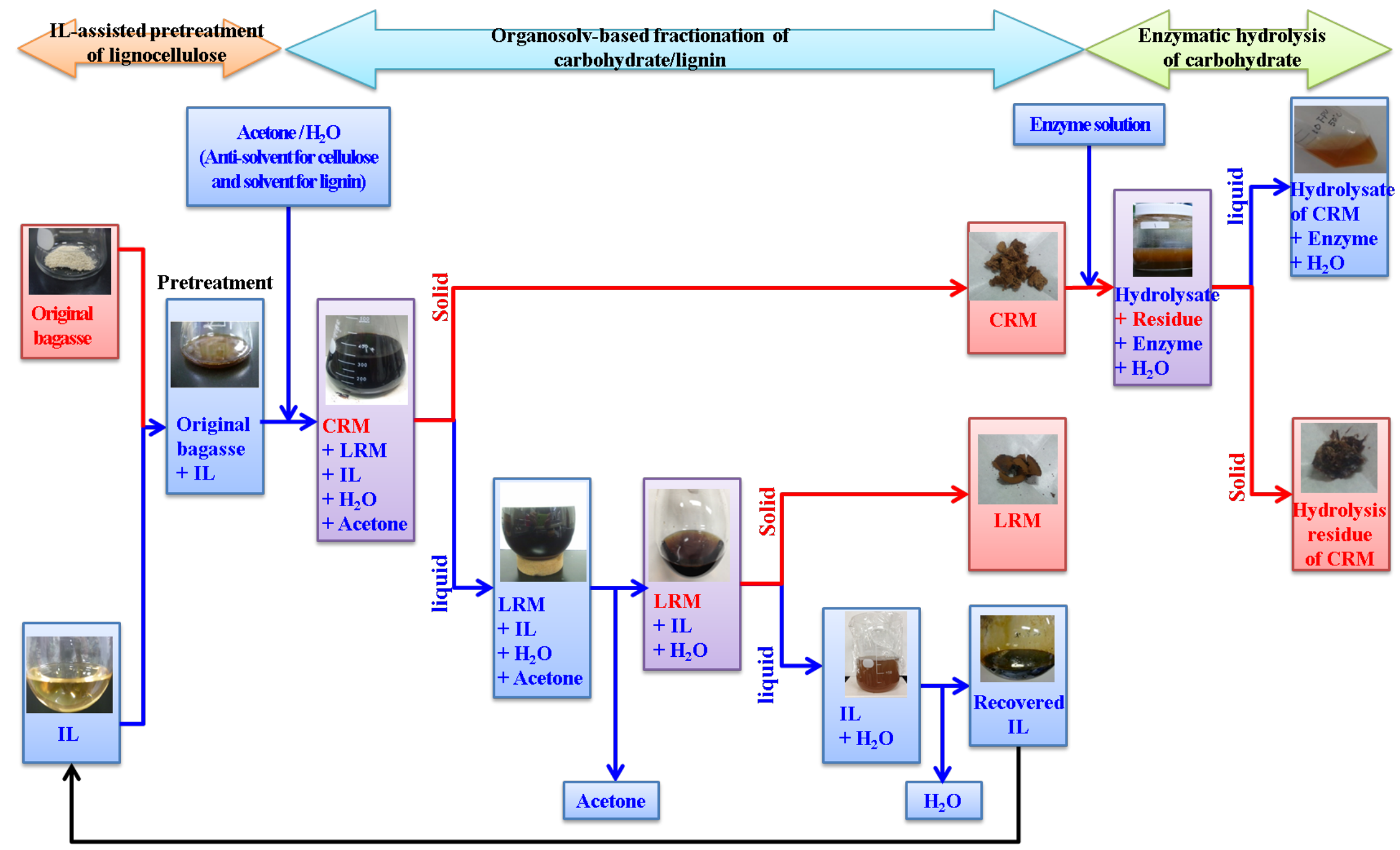

Fig. 1 Schematic diagram of IL-assisted pretreatment and subsequent fractionation of bagasse. 


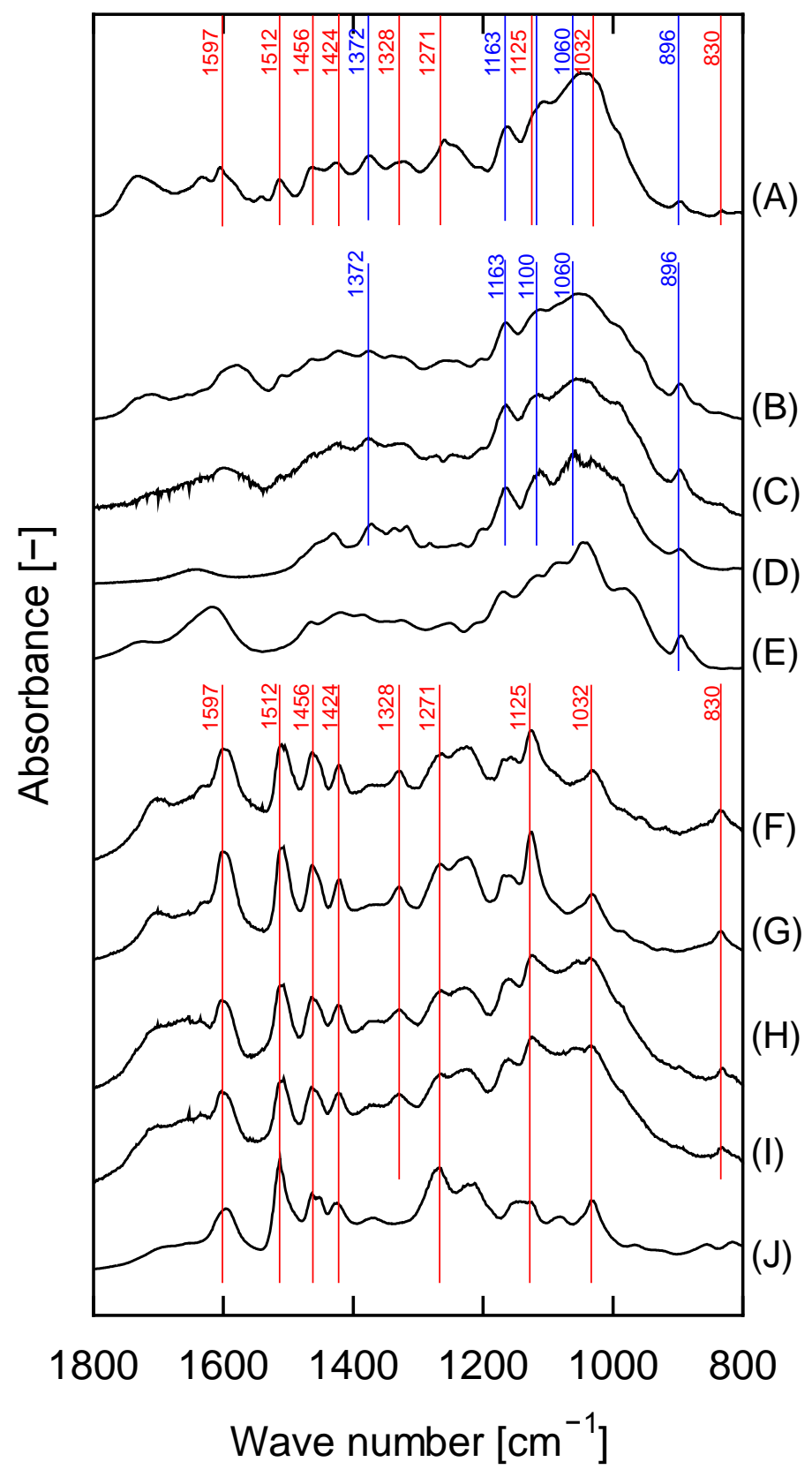

Fig. 2 FTIR spectra of cellulosic and lignin materials fractionated from bagasse. (A) original bagasse, (B) CRM from 1st cycle, (C) CRM from 5th cycle, (D) microcrystalline cellulose, (E) xylan, (F) LRM from 1st cycle, (G) LRM from 5th cycle, $(\mathrm{H})$ hydrolysis residue of CRM from 1st cycle, (I) hydrolysis residue of CRM from 5th cycle, and (J) alkali lignin. Vertical lines denote the characteristic peaks of cellulose/hemicellulose (blue lines) and lignin (red lines). 


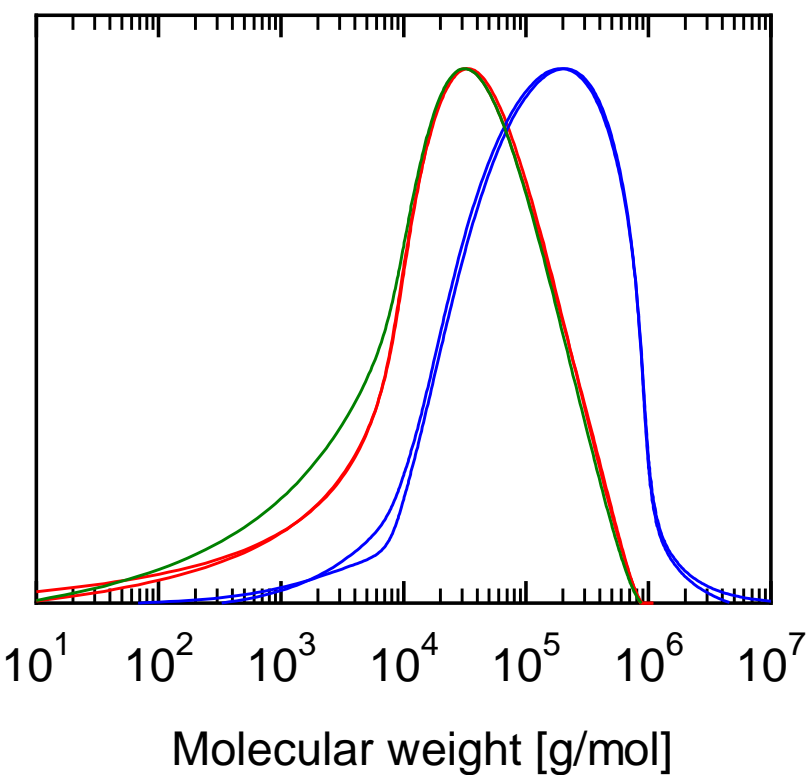

Fig. 3 GPC chromatograms of lignin materials fractionated from bagasse. Red lines: LRM from 1st cycle and 5th cycles. Blue lines: hydrolysis residue of CRM from 1st cycle and 5th cycles. Green lines: alkali lignin. 